\title{
Providers' Demeanor Impacts Patient Perceptions of Visit Length
}

\author{
Lauren C. Howe, PhD, Emerson J. Hardebeck, BA, Kari A. Leibowitz, MA, and Alia J. Crum, PhD
}

Department of Psychology, Stanford University, Stanford, CA, USA.

KEY WORDS: physician behavior; doctor-patient relationships; patientcentered care; patient engagement.

J Gen Intern Med 34(2):182-3

DOI: $10.1007 / \mathrm{s} 11606-018-4665-6$

(c) Society of General Internal Medicine 2018

\section{INTRODUCTION}

Time matters for healthcare quality. Patients explicitly desire more time with providers and patient perceptions of visit length influence meaningful outcomes, including patient satisfaction and adherence intentions. ${ }^{1,2}$ Given existing systemlevel barriers and competing demands (e.g., electronic health records, high patient volume), it may be challenging for providers to increase the objective amount of time they have with patients. ${ }^{3}$ However, research suggests that patient perceptions of time with the provider can outweigh actual visit length ${ }^{1,2}$ and, fortuitously, that these perceptions are malleable. ${ }^{4}$

Little research has tested what providers can do to shape patients' perceptions of the time providers spend with them. The current study tested whether provider demeanor, including the well-documented traits of warmth (e.g., attentiveness, care, and connection with patients) and competence (e.g., displays of knowledge, skill, and efficiency), ${ }^{5,6}$ influenced patient time perceptions of a fixed-length medical visit.

\section{METHODS}

To assess time perceptions in race/gender-concordant and discordant interactions, we recruited 187 White patients [64.2\% women, $\left.M(S D)_{\mathrm{Age}}=35.06(18.82)\right]$, who consented to undergo a standardized health screening to determine their eligibility for future health behavior studies with one of 13 randomly assigned providers of various races/genders [46.2\% women, 38.4\% Asian-American, 30.8\% African-American, $30.8 \%$ White]. Providers, who were medical or nursing students, were instructed to act in a professional manner but remain neutral with patients (e.g., minimizing small talk). Providers spent precisely $10 \mathrm{~min}$ with each patient, timed via stopwatch, administering a standardized medical protocol including health history and height, weight, and blood pressure measurements. Stanford University's IRB approved these procedures. Patients' cell phones were sequestered and exam rooms had no visible clocks to prevent awareness of actual time passed. Providers were blind to study hypotheses.

Published online September 13, 2018
Post-visit, patients rated provider warmth ( 8 items, $\alpha=.89$, e.g., "The medical practitioner was friendly," "...made me feel at ease") and competence (11 items, $\alpha=.92$, e.g., "The medical practitioner was intelligent," "...was skilled at the medical procedures") on 7-point scales ( $1=$ Strongly Disagree, $7=$ Strongly Agree) based on previous research ${ }^{5,6}$ that were averaged. Patients entered their estimation for minutes the provider spent with them in an open-ended text box. Providers were not present when patients completed these measures.

We used multiple linear regression $(R$ version 3.3.1, https:// www.R-project.org/) to predict time estimates with provider warmth and competence. Unstandardized regression coefficients were computed and $P$ values $\leq 0.05$ were considered statistically significant. Data are available from the corresponding author upon request.

\section{RESULTS}

Patients' perceptions of the 10-min encounter's length varied widely; estimates ranged from 2 to $30 \mathrm{~min}$ (Fig. 1) $(M(S D)=$ 11.96(5.69)). Provider warmth predicted longer perceived visit time $(B=1.26$ [95\% CI, 0.23 to 2.30$], S E=0.52, P=0.017)$ (Fig. 2). Each additional point in rated warmth $(M(S D)=$ $5.08(0.98)$ ) increased perceived visit length by approximately $1.26 \mathrm{~min}$, corresponding to a 5.06-min difference between the lowest and highest warmth ratings ( 3 and 7 on the 7-point scale, respectively). Results remain significant when accounting for provider race and gender (both non-significant covariates). Results remain significant when omitting outliers $(N=7$ patients who estimated a 30-min visit, $+3 S D$ s above the mean). Provider competence did not predict longer perceived visits $(B=0.31$ [95\% CI, -0.97 to 1.896$], S E=0.74, P=0.50)$.

\section{DISCUSSION}

This study highlights provider demeanor as an underexplored, cost-effective avenue for maximizing the limited time providers have with patients. Across highly standardized, timed interactions with diverse providers, patients who rated providers as warmer (i.e., more attentive, caring, and connected) believed these providers spent more time with them. The 5.06-min increase in perceived visit length between the most and least warmly rated providers corresponds to a 31.6 to $38.9 \%$ increase in perceived length for the median US office visit (13-16 min). 


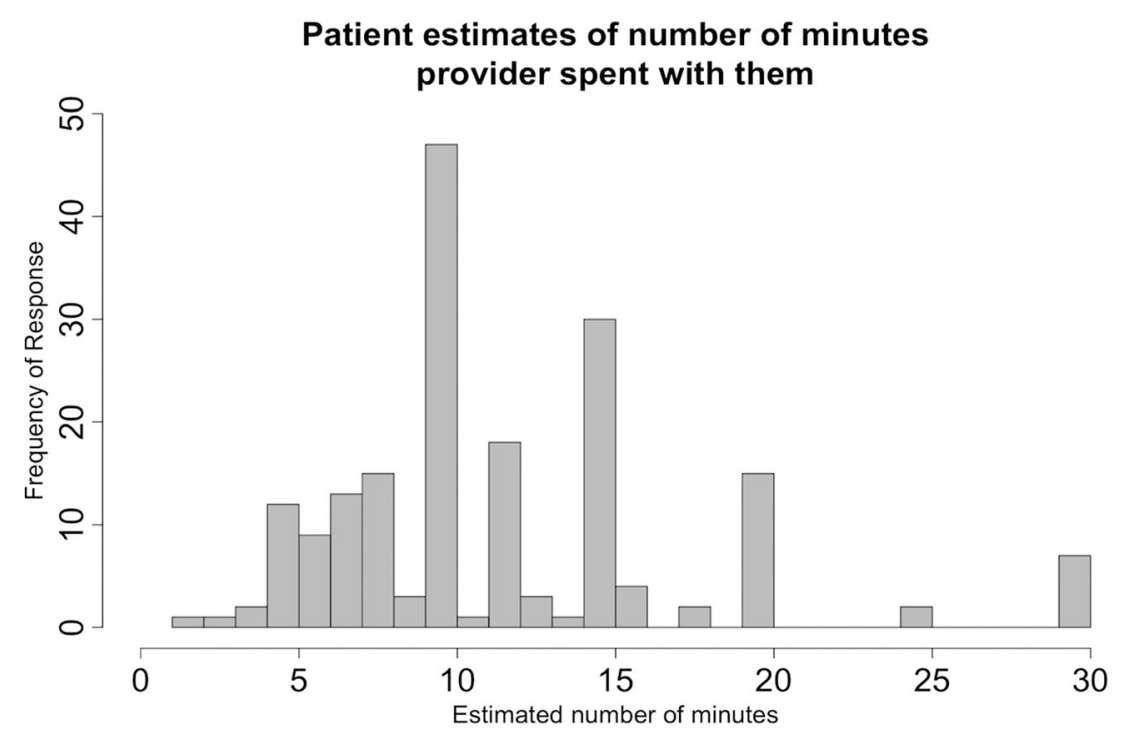

Figure 1 Patient estimates of visit length (in minutes).

While using a physical examination for this study allowed for consistently timed, highly controlled interactions, future research should be conducted in diverse clinical contexts to further assess the causal relationship between perceived warmth and time and the downstream effects of time perceptions on patient outcomes.

These results contribute to the science, practice, and business of medicine, suggesting a low-cost way to bolster perceived visit length without necessitating additional time. System-level changes to increase actual time with patients are clearly needed. However, increasing perceived time is an underutilized route

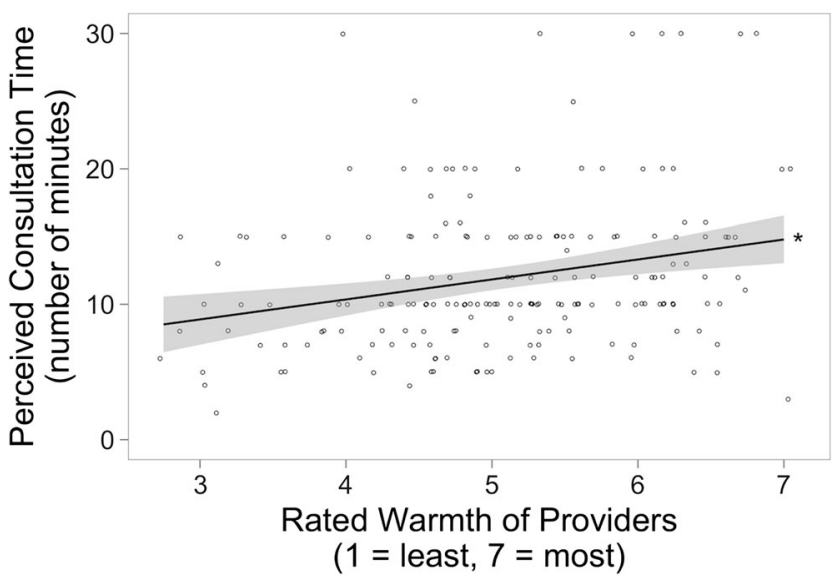

Figure 2. Associations of ratings of provider warmth with perceived length of visit time. The line represents the relationship between provider warmth and estimated minutes of visit length (shaded region $=95 \%$ confidence interval). Points represent patients' individual responses. Multiple linear regression was used to test the significance of the predictors. $* P<0.05$. to improving patient experience. Research suggests that perceptions of provider warmth are malleable. ${ }^{5}$ By signaling warmth during the clinical encounter, providers may change perceptions of visit length to improve medical care.

Contributors: We would like to acknowledge Michelle Chang and Isaac Handley-Miner for their invaluable assistance with this project.

Corresponding Author: Lauren C. Howe, PhD; Department of Psychology Stanford University, Stanford, CA, USA (e-mail: Lchowe@stanford.edu).

Funders This research was supported by the Robert Wood Johnson Foundation.

\section{Compliance with Ethical Standards:}

Stanford IRB approved these procedures.

Conflict of Interest: The authors declare that they do not have a conflict of interest.

\section{REFERENCES}

1. Cape J. Consultation length, patient-estimated consultation length, and satisfaction with the consultation. Br J Gen Pract. 2002;52(485):1004-6.

2. Swayden KJ, Anderson KK, Connelly LM, Moran JS, McMahon JK, Arnold PM. Effect of sitting vs. standing on perception of provider time at bedside: A pilot study. Patient Educ Couns. 2012;86(2):166-71.

3. Sinsky C, Colligan L, Li L, Prgomet M, Reynolds S, Goeders L, et al. Allocation of physician time in ambulatory practice: A time and motion study in 4 specialties. Ann Intern Med. 2016;165(11):753-60.

4. Park C, Pagnini F, Reece A, Phillips D, Langer E. Blood sugar level follows perceived time rather than actual time in people with type 2 diabetes. Proc Natl Acad Sci. 2016;113(29):8168-70.

5. Howe LC, Goyer JP, Crum AJ. Harnessing the placebo effect: Exploring the influence of physician characteristics on placebo response. Heal Psychol. 2017;36(11):1074-82.

6. Fiske ST, Cuddy AJC, Glick P. Universal dimensions of social cognition: warmth and competence. Trends Cogn Sci. 2007;11(2):77-83. 